\title{
Integration of Relational and NoSQL Databases
}

\author{
Jaroslav Pokorný \\ Faculty of Mathematics and Physics \\ Charles University \\ Malostranské nám. 25 \\ 11800 Praha 1, Czech Republic \\ pokorny@ksi.mff.cuni.cz
}

Received 9 May 2019

Accepted 30 June 2019

Published 24 July 2019

\begin{abstract}
The analysis of relational and NoSQL databases leads to the conclusion that these data processing systems are to some extent complementary. In the current Big Data applications, especially where extensive analyses (so-called Big Analytics) are needed, it turns out that it is nontrivial to design an infrastructure involving data and software of both types. Unfortunately, the complementarity negatively influences integration possibilities of these data stores both at the data model and data processing levels. In terms of performance, it may be beneficial to use a polyglot persistence, a multimodel approach or multilevel modeling, or even to transform the SQL database schema into NoSQL and to perform data migration between the relational and NoSQL databases. Another possibility is to integrate a NoSQL database and relational database with the help of a third data model. The aim of the paper is to show these possibilities and present some new methods of designing such integrated database architectures.
\end{abstract}

Keywords: Relational database; NoSQL database; Big Data; Big Analytics; database integration.

\section{Introduction}

Recently, most large enterprises seem to be actually taking care of minimizing the application maintenance of existing production systems. This causes "bad" database schemas to be used and "database decay" generally occurs. The authors of Ref. 1 built on discussions with nearly 20 database administrators (DBAs) at three very large enterprises:

- The databases vary depending on the business conditions, usually once a quarter or more.

This is an Open Access article published by World Scientific Publishing Company. It is distributed under the terms of the Creative Commons Attribution 4.0 (CC BY) License which permits use, distribution and reproduction in any medium, provided the original work is properly cited. 
- The environment leads to the often disappearing central DBA's roles and a more decentralized approach with more DBA groups maintaining databases in the enterprise.

- NoSQL databases, a database alternative for storage and processing so-called Big Data today, contribute to this state significantly.

The history of database management system (DBMS) always reflected the requirements concerning new types of data to be stored in a database way. Several database models such as object-oriented (OO), object-relational (OR), XML or RDF have been introduced since the relational data model was introduced. OO and OR DBMSs responded to object-oriented approaches to software engineering from the 1990s. However, these tools have never been competitive in the market. Reasons might be due to the lack of their theoretical foundations and the limited performance in practice. The XML databases suffer from similar problems. Their goal is to promote the distribution of XML documents, but the use of native XML databases is rather limited. Major vendors of relational DBMSs (RDBMSs), such as Oracle, Microsoft SQL Server and MySQL, include XML support in their products, but native XML databases are not much involved in the database market. The initial enthusiasm for XML databases was based on Web application architectures and service orientations that use XML as a means to standardize data exchange format. However, this is now already possible with document-oriented NoSQL databases (see the popular JSON ${ }^{\mathrm{a}}$ format), though not in such powerful languages as the XQuery in XML environment. However, the XML format has been added to the relational environment and is now the basic data type in SQL databases.

The situation in the database world today is affected by Big Data. Their $V$ characteristics are Volume, Velocity and Variety. The author of Ref. 2 lists even 11 such $V$ s. They fundamentally affect the storage and processing infrastructure of Big Data. Effective use of systems involving the processing of large volumes of data requires, in many application scenarios, adequate tools for storing and processing such data at a low level and analytical tools at higher levels. In other words, Big Data is an evolving term that describes a large volume of structured, semi-structured and unstructured data that have the potential to be mined for information and used in machine learning (ML) projects and other advanced analytics applications. Thus, from the user's point of view, the most important aspect of processing large volumes of data on a computer is their analysis, as it is now called Big Analytics.

Unfortunately, large data collections include data in different formats, such as relational tables, XML data, text data, multimedia data or RDF triples, which may cause problems in processing data mining (DM) algorithms. Also, the growing data volume in a repository or the number of users of this repository requires a reliable solution of scaling in these dynamic environments, and more advanced means of delivering high performance than offered by the traditional database architectures. Moreover, traditional RDBMSs lack the dynamic data model necessary to tackle

a https://www.json.org/. 
high-velocity data coming in from machine-generated systems or time-series applications, as well as cases needing to manage social media data.

It is obvious that Big Analytics is also performed over a large amount of transaction data by extending the methods commonly used in data warehouses (DWs). But DW technology has always been focused on structured data compared to the much richer variability of data types, as it is today usual for Big Data. Analytical processing of large data volumes therefore requires not only new database architectures but also new methods for data analysis. On the other side of data storages with well-defined structures, we can find the so-called data lakes now. A data lake is a storage repository that holds a vast amount of raw data in its native format until it is needed. The data lake can also act as an online archive for infrequently accessed data. While DWs store data in files or folders, a data lake uses a flat architecture to store data. Each data element in a lake is assigned a unique identifier and tagged with a set of extended metadata tags. Because the data in a lake can often originate from sources outside of the company's operational systems, lakes are not a good tool for usual business analytics user.

In the ICT history, different DBMSs were designed to solve different problems, considering still new and new data types. In addition to centralized RDBMSs, specialized servers, universal servers, relational DW, etc. appeared in the past. These tools were based on a fixed database schema and an associated query language (mostly SQL). OR SQL and its other extensions supported this strategy for a long time.

To store and process Big Data today, we can choose from many options:

- traditional DBMS (hereinafter referred to as databases or shortly DBs) - relational (SQL), OO and OR;

- traditional parallel database systems ("shared-nothing" architectures);

- distributed file systems (e.g. HDFS);

- NoSQL databases;

- NewSQL databases;

- integrated database architectures; and

- clouds.

Now, in usual practice, ITC and business professionals need to determine whether NoSQL technologies are better suited than RDBMS for a particular system. The choice of technology is critical for applications that can be both transactional and analytical. These technologies typically require different software and hardware architectures. The aim of this paper is to discuss the relation between SQL databases and NoSQL databases, modeling databases in the SQL and NoSQL polyglot world, in order to support Big Analytics. An attention is devoted to problems of integration of such heterogeneous platforms in one architecture.

This work is an extended version of the conference paper. ${ }^{3}$ In Sec. 2, we briefly describe the Big Analytics concept, i.e. the properties of processing and analysis of large volumes of data. In Sec. 3, we briefly review the NoSQL database technologies, 
especially their data models, architectures and some of their representatives. We show the duality between SQL databases and NoSQL databases. Section 4 presents various integrated database architectures composed of both NoSQL databases and a mix of RDBMS and NoSQL. Section 5 contains conclusions and challenges for the database community.

\section{Analytical Processing of Big Data}

Big Analytics is used to transform information into knowledge through a combination of existing and new approaches. Related technologies include:

- data management (considering uncertainty, real-time query processing, information extraction and explicit time dimension management);

- new programming models;

- statistical methods, DM and ML;

- component architectures of data storage and processing systems; and

- visualization of information.

As usual, two types of data processing are distinguished:

- real-time processing (data-in-motion) concerns a stream of data that is moving or being transported between locations or between computer systems,

- batch processing of data obtained from different sources into one database (dataat-rest) followed by analysis after the events occur.

Batch analysis can then be:

- small (Small Analytics), i.e. OLAP over DW,

- $b i g$ (Big Analytics), i.e. both DM and ML.

The problems that arise in this context are based on the fact that the requirements for Big Data are often more dynamic than the classic data processing in DWs. This concerns all three $V$ s mentioned in Sec. 1.

A volume is not only a problem for data storage but also influences Big Analytics. With the increase in data complexity, its analysis is also more complex. We need to scale both the infrastructure and the standard data processing techniques for Big Data. Speed can also be a problem because the value of the analysis (and often of the data) decreases over time. If multiple data stream passes are required, data must be entered into DW where further analysis can be performed. Data can thus be stored and processed in a relatively traditional way or using cheap systems such as distributed NoSQL database. The NoSQL database is an alternative. Another issue is how to analyze Big Data coming from relational DBs, possibly from databases of both types, i.e. from relational and NoSQL ones.

Big Data is often mentioned only in relation to business intelligence (BI). However, not only BI developers but, generally, data scientists also analyze large data collections. The challenge for computer professionals or data scientists is to provide people 
with tools that can efficiently perform complex analytics, taking into account the particular nature of processing large volumes of data. It is important to emphasize that Big Analytics does not only include analysis and modeling phases. Often, distorted context as well as data heterogeneity and interpretation of results are taken into account. All these aspects affect scalable strategies and algorithms, so more efficient preprocessing steps (filtering and integration) and advanced parallel computing environments are needed. Data variability is now part of Big Data storage design and analytical system design. But performance is still a first-order requirement.

In addition to these rather classic issues of mining large volumes of data, other interesting issues have emerged in recent years, such as recognizing named entities. ${ }^{4}$ The analysis of views and opinions (such as positive, negative or neutral) and their mining (sentiment analysis) are actual as the topics using information retrieval methods and Web data analysis. A specific problem is the search for and characterization of discrepancies based on views and opinions. Comparison of graph patterns is commonly used in social network analysis where graphs, for example, include a billion users and hundreds of millions of links. In any case, the main problems of the current DM techniques used for Big Data come from their lack of scalability and parallelization.

\section{NoSQL Databases}

Large-scale data collections are often used for the storage and processing in NoSQL databases. NoSQL means "not only SQL", which makes this database category very diverse and not very clearly specified. NoSQL databases, starting in the late 1990s, provide easier scalability and performance compared to traditional RDBMS. We briefly describe their properties and classification (Sec. 3.1), followed by a discussion of their usability (Sec. 3.2). A more detailed discussion of NoSQL databases and, more generally, their relationships to Big Data issues can be found, for example, in Refs. 2 and $5-7$.

\subsection{Categories of NoSQL databases}

What is the main classical approach to databases - a (logical) data model — is described in NoSQL databases rather intuitively, without any formal basis. NoSQL terminology is also very diverse, and the difference between conceptual and database views is mostly blurred.

The most well-known NoSQL databases can be classified according to the used data model as:

(1) key-value stores, such as Redis ${ }^{\mathrm{b}}$;

(2) column stores, e.g. Cassandra ${ }^{c}$; and

(3) document stores, such as MongoDB. ${ }^{\mathrm{d}}$

\footnotetext{
b https://redis.io/.

chttp://cassandra.apache.org/.

d https://www.mongodb.com/.
} 
Key-value stores contain a set of pairs (key, value). The key uniquely identifies the opaque value. The choice of the key is, unlike relational databases, solved pragmatically. The goal is only quick access to data. The value can even be a list of pairs (name,value) (e.g. in Redis). Data access operations, typically get and put, only work through the key. These stores have no implicit ordering, but some implementations allow to order the database by the value of the key, thereby enabling range searches against the key. Although it is a very efficient and scalable approach for implementation, the disadvantage of a too simple data model can be substantial for such DBs. However, NULL values are not required, because these databases do not use the schema.

Column stores or wide-column stores can contain a set of couples (name,value) in a column family in a row addressed by a key. A column family in different rows can contain different columns. Each row actually has a relatively small number of columns compared with the total number possible. This arrangement remains as a sparse matrix. Often we are talking about a column-oriented NoSQL database. There is also another level of structure called a supercolumn in Cassandra. The supercolumn contains nested (sub)columns. Access to data using get and put is enhanced by using column names.

Most general NoSQL data models belong to document stores. They are the same as key-value stores, but each key is coupled with any complex data structure that resembles a semi-structured document. The JSON format is usually used to present these data structures. JSON is a typed data model that supports basic data types and objects - nonordered sets of couples (name,value), and the value can be structured (array). JSON is similar to XML, but it is smaller, faster and easier for parsing. For example, CouchDB ${ }^{\mathrm{e}}$ uses the JSON format whereas MongoDB stores data in BSON (binary-coded serialization of JSON documents) format. It is possible to query the data in a document in other ways than using a key (e.g. through indexing), i.e. we can obtain only portions of the document as the query result. Moreover, selection and projection operations on the query results can be performed.

Without doubts, these data models can be generalized to two concepts (key and value), which is a property usable for integration of NoSQL DB (see Sec. 4.3.1).

There are also other approaches in the NoSQL world. DB-Engines Ranking server, ${ }^{f}$ for example, considers also search engines as NoSQL databases, e.g. Elasticsearch. ${ }^{\mathrm{g}}$ They are DBMSs dedicated to the search for data content. They are not typically classical document systems. They usually offer a support for complex search expressions, full-text search, ranking and grouping of search results, geospatial search and distributed search for high scalability. More generally, NoSQL databases include also graph databases ${ }^{8}$ and others, e.g. XML and RDF ones.

Often, a question is whether OR database is also a NoSQL database. The answer is no, because NoSQL databases are normally denormalized (save copy of object data

e http://couchdb.apache.org/.

$\mathrm{f}_{\text {https: } / / \text { db-engines.com/en/ranking. }}$

g https://www.elastic.co/products/elasticsearch. 
in place of object), whereas OR database is a normalized database with object relationships.

We can observe that the NoSQL categories (1)-(3) are basically of the key-value type. They differ mainly in the possibilities of aggregating couples (key,value) and accessing these values. For our considerations, we consider only them.

\subsection{Usability of NoSQL databases}

There is much debate about the role of NoSQL databases in providing information services. NoSQL promoters claim that this technology is the future of databases. On the other hand, the RDBMS supporters argue that the NoSQL databases have a big disadvantage of failing to provide correct data integrity. In any case, NoSQL technologies being designed with Big Data needs to be kept in mind.

NoSQL-based applications are often a part of data-intensive cloud applications (mainly Web applications). Examples of such applications include Web entertainment applications, high-traffic website services, media delivery in a streamlined fashion or typical data found in social networking applications.

NoSQL systems are more suitable for interactive data services environments. Schema enforcing and row-level locking as in relational databases may overcomplicate these applications. The absence of some ACID properties even allows significant acceleration and decentralization of NoSQL databases.

On the other hand, one of the most famous problems with NoSQL repositories is the lack of semantics caused by their underling feature - they are schemaless. The lack of metadata prevents the database system from knowing which data is stored and how it is interconnected.

NoSQL databases usually have little means of ad-hoc querying and analysis. Even a simple query requires significant programming experience, and the generally used BI tools do not provide connectivity to NoSQL. NoSQL databases cannot also be recommended for applications requiring enterprise-level functionality (ACID properties, security and other relational technology features). In any case, NoSQL should not be the only choice in the cloud.

Experience with the NoSQL databases shows that they can be used even on "small" data. They are appropriate for applications not requiring transactional semantics, such as directories, blogs or content management systems, or for analyzing high-volume, real-time data (e.g. website click-streams). We remind that in the mobile data processing environment, transactions are even more technically impossible in a larger range.

Among the good properties of NoSQL databases we can find:

- massive performance of write operations;

- quick search in a key-value way;

- they do not have any portion causing a total network failure when an error occurs;

- they enable rapid prototyping and development; 
- they allow scalability without user intervention; and

- they allow easy maintenance.

On the other hand, a user may find unusual and often inappropriate phenomena in NoSQL approaches:

- they show different behavior in different applications;

- a complete and fully formalized data modeling procedure for NoSQL DB does not exist;

- no language query standards are available. Typically, users must interact with these systems at a programming level with custom APIs. This reduces portability and requires system-specific code;

- migration from one system to another is complicated;

- join operation is missing;

- some of them are more mature than the others, but each of them solves similar problems;

- checking referential integrity "over" database partition segments is missing. As the performance is crucial, an integrity control or the implementation of complex operations is limited in a distributed environment.

Table 1 shows a comparison of NoSQL databases and RDBMSs in more detail. Particular facts contained there also reveal some basic properties of the considered types of DBMSs, e.g. horizontal scaling (also called sharding) of NoSQL ones involves partitioning the database on the value of some field.

Table 1. Comparison of relational and NoSQL DBs.

\begin{tabular}{|c|c|c|c|}
\hline & Property & SQL & NoSQL \\
\hline 1 & Data model & relational & domain \\
\hline 2 & & data is strongly typed & $\begin{array}{l}\text { data is potentially dynamically } \\
\text { typed }\end{array}$ \\
\hline 3 & & $\begin{array}{l}\text { data of dependent tables point to } \\
\text { their parents (via foreign keys) }\end{array}$ & $\begin{array}{l}\text { parent's data point to children's } \\
\text { data }\end{array}$ \\
\hline 4 & & $\begin{array}{l}\text { entities have an identity (primary } \\
\text { key) }\end{array}$ & environment determines identity \\
\hline 5 & & not compositional & compositional \\
\hline 6 & & $\begin{array}{l}\text { referential integrity based on } \\
\text { values }\end{array}$ & $\begin{array}{l}\text { weak referential integrity based on } \\
\text { computation or only "over" } \\
\text { partition segments }\end{array}$ \\
\hline 7 & Integrity & responsibility at the DB level & $\begin{array}{l}\text { responsibility moved to the appli- } \\
\text { cation level }\end{array}$ \\
\hline 8 & DB schema & schema expressed in SQL & $\begin{array}{l}\text { typically do not require a fixed DB } \\
\text { schema, i.e. they have a more } \\
\text { flexible data model }\end{array}$ \\
\hline 9 & $\begin{array}{l}\text { Detection of problems in } \\
\text { the DB schema }\end{array}$ & at the DB level & $\begin{array}{l}\text { at the application level and data } \\
\text { access procedures }\end{array}$ \\
\hline 10 & $\begin{array}{l}\text { DB modeling } \\
\text { management }\end{array}$ & begins from accessible data & $\begin{array}{l}\text { patterns for data access and } \\
\text { updates }\end{array}$ \\
\hline
\end{tabular}


Table 1. (Continued)

\begin{tabular}{|c|c|c|c|}
\hline & Property & SQL & NoSQL \\
\hline 11 & Querying & SQL & $\begin{array}{l}\text { simple API, if SQL, then only its } \\
\text { very limited version; REST, } \\
\text { client libraries }\end{array}$ \\
\hline 12 & & complex queries $+a d-h o c$ queries & $\begin{array}{l}\text { inappropriate for } a d-h o c \text { queries } \\
\text { and complex queries }\end{array}$ \\
\hline 13 & & join operation & $\begin{array}{l}\text { join emulation at the application } \\
\text { level }\end{array}$ \\
\hline 14 & Data storage & centralized or distributed & horizontally scaled, replications \\
\hline 15 & Data processing & $\begin{array}{l}\text { synchronous (ACID) updates over } \\
\text { more rows }\end{array}$ & $\begin{array}{l}\text { asynchronous (BASE) updates } \\
\text { within single values }\end{array}$ \\
\hline 16 & & $\begin{array}{l}\text { environment coordinates change } \\
\quad \text { (transactions) }\end{array}$ & $\begin{array}{l}\text { entities responsible for reacting to } \\
\text { changes (eventual consistency) }\end{array}$ \\
\hline 17 & & $\begin{array}{l}\text { strong consistency and also the } \\
\text { consistency is tunable by } \\
\text { application }\end{array}$ & $\begin{array}{l}\text { eventual and also the consistency is } \\
\text { tunable in application }\end{array}$ \\
\hline 18 & & $\begin{array}{l}\text { query optimizer - responsibility } \\
\text { of DBMS }\end{array}$ & $\begin{array}{l}\text { developer/pattern - responsibility } \\
\text { of application }\end{array}$ \\
\hline
\end{tabular}

In the database world NoSQL databases occupy a significant place. In the DB-Engines Ranking, 347 various DB-Engines were tracked in May 2019. MongoDB, Redis and Cassandra occupy the positions 5, 8 and 10, respectively, in this rating.

\section{SQL and NoSQL: Towards Integrated Architecture}

In the work, ${ }^{9}$ the authors argue that the NoSQL databases are rather complementary to traditional transactional DBMSs. Should not they be called "corelational"? Maybe more natural would be to say coSQL instead of NoSQL. In Table 1, according to Ref. 9, complementary differences are given by the properties 2-6, 14, 15 and 17.

Particularly, normalization allows single object data in a relational database to be spread over multiple relations. For example, customer data is in one table, data about the banks where the account exists is in the second table. The interconnection is made via foreign keys. In a NoSQL database, this can be done in such a way that, for example, each bank "row" can contain the data and account numbers for each customer. The basic feature of NoSQL is that they are denormalized, i.e. they store copies of an object instead of the object. This, of course, leads to worse data update options but to more effective querying. Consequently, this complementarity negatively affects integration possibilities of these data stores both at the data model and data processing levels.

In Sec. 4.1 we describe some general approaches to database integration. Then we focus on two most occurring categories of these approaches: multimodel approach (Sec. 4.2) and multilevel modeling (Sec. 4.3). 


\subsection{Approaches to integration}

Concerning an integration of distributed data from different databases, two approaches based on a database schema management were at disposal:

- top-down: This approach starts with a global schema to design schemas for data in sites;

- bottom-up: This approach is through middleware, i.e. to use schema mapping for schemas in sites onto a middleware (e.g. OLE database, JDBC) and then use a query transformation. Data is loosely integrated and managed by multiple servers.

We remind that the former concerns rather homogenous database models, while the latter supports heterogeneous database models and consequently heterogeneous DBMSs. Thus, the latter variant will be relevant here.

In the context of RDBMSs and NoSQL databases, it is not possible to use simply traditional approaches to data integration. The reason is the complementarity of these database types. Moreover, the problem of analysts is that the lack of data schemas (semantics) prevents them from understanding their structure and thus generating serious analyses. Now, the tendency is to create multimodel and multilevel modeling approaches involving both relational and NoSQL architectures including their integration. ${ }^{10}$ Moreover, data modeling (or schema design) is in fact more important when dealing with NoSQL than it was with relational databases. We just need a different kind of data modeling than in the past. Since NoSQL database does not have schema, data schema needs inference from raw data, instead of being extracted directly from the database like RDBMS. ${ }^{11}$

We mention also the so-called polyglot persistence in this context. Here, we approach particular data stores with their original data access methods. ${ }^{12}$ The truth is that polyglot persistence is a method for data modeling problems, not a solution to them. It means that in practice we may have a different database for every application or even a bunch of different databases for a single application. Developers need to customize data models for an application and often need more than one, but they should not have to adopt different DBMSs to get them. "Polyglot" means "able to speak many languages", not integration, i.e. cross-database queries are very hard to implement. This way to database integration is sometimes called single-model polyglot persistence approach. As an integration architecture, single polyglot persistence is its weakest form. Recently, a more adequate alternative to fulfill the polyglot persistence requirement is a new class of data store systems that integrates several NoSQL data models, leading to the so-called NoSQL multimodel databases. These systems simplify the process of application development, but at the same time, they can decrease the performance of applications. ${ }^{13}$

An interesting variant occurs in the case when relational database and NoSQL database have to co-exist. In practice, there are options, such as the schema conversion model, in which the schema from the SQL database is converted to the 
NoSQL database schema. ${ }^{14}$ Then, even a double-sided data migration between an RDBMS and a NoSQL database can be performed.

\subsection{Multimodel approach}

Maybe, multimodel approach presents a more user-friendly solution of heterogeneous database integration. Vendors have mixed and matched elements from different NoSQL databases to achieve more generally useful systems. Multimodel represents an intersection of multiple models in one product. Not only NoSQL stores participate in such integrated architecture, sometimes NoSQL elements are mixed with SQL elements. The category of such integrated databases, i.e. not only NoSQL ones, is referred to as multimodel databases. For example, there are a lot of products in this DBMS category, as follows:

- OrientDB ${ }^{\mathrm{h}}$ is a multimodel DBMS including geospatial, graph, full-text and keyvalue data models. OO concepts are used for user domain modeling in OrientDB. The basic (the smallest) data unit is a record. There are four possible record types: byte record (BLOB), document, node and edge. Document is the most flexible type of record because it can be schemaless. Records are grouped into clusters.

- ArangoDB ${ }^{\mathrm{i}}$ is designed as a native multimodel database, supporting key-value, document and graph data models. A document can consist of any number of attributes with values (of simple or complex type). There are two kinds of collections in ArangoDB: document (nodes) collection and edges collection. Edge is a special kind of document which has two peculiar attributes: _from and _to are used to denote the relation between documents. So documents are organized into a directed graph.

- Couchbase Server ${ }^{\mathrm{j}}$ supports both key-value and document approaches. Couchbase Server uses the memcached binary protocol for key-value operations and REST APIs for N1QL and view queries. N1QL is a powerful declarative language for querying, transforming and manipulating JSON data.

- Apache CouchDB stores JSON documents, with the option of attaching nonJSON files to those documents. It uses a MapReduce pattern to index data. Queries in CouchDB pull data from what are essentially stored procedures called views. A view is made up of a map function and optionally a reduce function.

- DataStax Company offers DataStax Enterprise ${ }^{\mathrm{k}}$ (DSE) platform to meet the multimodel needs. DSE built on the Apache Cassandra supports key-value and tabular data models, JSON/document model and graphs.

\footnotetext{
${ }^{\mathrm{h}}$ http://orientdb.com/orientdb/.

i https://www.arangodb.com/.

j https://www.couchbase.com/products/server.

k http://www.datastax.com.
} 
- MarkLogic ${ }^{1}$ enables to store and search JSON and XML documents and RDF triples. It can also store images, videos and more. The database is not only scalable, but also has enterprise security and ACID transactions.

Obviously, implementations of such approaches are different in general. For example, OrientDB and ArangoDB implement the graph engine using different data structures.

A co-existence of RDBMS and a NoSQL database is denoted sometimes as NoSQL-on-RDBMS approach. It includes, for example, storing and querying JSON data in an RDBMS (see ARGO/SQL ${ }^{15}$ ) or Oracle 19c DBMS supporting JSON documents, spatial and graph data, text and XML data and multimedia data that can be used and managed inside Oracle database. We remind that various standard extensions of SQL language enable this approach. Oracle mentions the so-called multimodel polyglot persistence in this context.

NoSQL multimodel databases are classified to single-store multimodel DBMSs (denoted hereafter for simplicity as multimodel DBMSs) and tightly integrated polystores. ${ }^{16}$ The former means that a multimodel DB is designed to support multiple data models against a single, integrated backend. Polystores (also called multistore systems) provide integrated or transparent access to a number of cloud data stores through one or more query languages. ${ }^{17}$ More generally, multiple data storage technologies are chosen based on the way data is being used by individual applications. The work ${ }^{18}$ offers an effective integration approach of relational DBs and NoSQL data stores, including MySQL, MongoDB and Redis, which allows users to query data from both relational SQL systems and NoSQL systems in a single SQL query.

\subsection{Multilevel modeling}

Despite the fact that DB schemas are mostly not used in the NoSQL world, some variations on multilevel modeling approaches exist. Usually the data interoperability of NoSQL systems is increased. In relation to solution of an alternative for data processing with relational and NoSQL data in one infrastructure, the common design methods for such DBs are based on the modification of the traditional three-level ANSI/SPARC approach. ${ }^{19}$ The approach involves not only heterogeneous data sources but also the development of a database schema in the overall infrastructure, i.e. its variability. Multilevel models describe a system at least at two different levels. Interactions take place within and between those levels. A strong motivation for this approach is the fact that when designing a database for Big Analytics, we must consider DM/ML patterns, clustering of some attributes, etc. to ensure adequate system performance. However, the conceptual design assumes the correctness of the current knowledge of the application domain. Only few approaches exist to query data from NoSQL and relational database systems simultaneously. The following

${ }^{1}$ http://www.marklogic.com/. 
examples document activities in this area: rather simple approach called NoSQL relationally (Sec. 4.3.1) and more advanced integrating approaches using a special abstract model (Sec. 4.3.2) or, even, ontologies (Sec. 4.3.3).

\subsubsection{NoSQL relationally}

Architectures used in this category of databases contain one or more NoSQL databases. Some approaches have been proposed for providing mapping of relational DB schemata and operations onto equivalent ones in NoSQL databases to deal with large relational datasets in the cloud, focusing on scalability and availability.

- Authors of Ref. 20 present the SQLtoKeyNoSQL, a layer able to translate relational schemata as well as SQL commands to equivalent schemata and access methods to any key-oriented NoSQL databases [see (1)-(3) from Sec. 3.1]. A canonical data model serves as an intermediate data model between the relational data model and all key-based access NoSQL data models, providing schema mapping flexibility and access transparency to the applications.

- The multimodel solution ${ }^{21}$ considers source document and column-oriented database integrated through a middleware into a virtual SQL database. Its authors propose a Bridge Query Language (BQL) that enables a transformation from an SQL query defined over the target to the query executed over a given source. The results obtained from each query are later processed within the data integration system.

- The work $^{22}$ presents the JackHare framework with SQL query compiler, JDBC driver and a systematical method using MapReduce framework for processing unstructured data in column-oriented key-value store HBase. ${ }^{\mathrm{m}}$ MapReduce jobs take advantage of parallel processing for improving the join operation performance.

\subsubsection{Special abstract model}

- A database design methodology for NoSQL systems based on NoSQL Abstract Model (NoAM), a novel abstract data model for NoSQL databases, is presented in Ref. 23. The methodology starts with a UML class diagram, a designer identifies the so-called aggregates ("chunks" of related data) and maps the aggregates into NoAM blocks. These blocks are simply transformed into constructs of a particular NoSQL data model.

- Data integration method presented in Ref. 11 is based on JSON objects and it allows to resolve both semantic and syntactic heterogeneities of source systems. Results of source database queries are translated to JSON objects, and finally data merging is done by concatenating separate JSON files. The solution does not support joins and aggregates across data sources; it only collects data from

$\mathrm{m}_{\text {http://hbase.apache.org/. }}$ 
different separated DBMSs according to the filtering options and migrates them. A metamodel approach is used here as well as a graphical query interface.

- Authors of Ref. 24 propose Heterogeneous Distributed Model (HerM), a NoSQL data modeling approach which supports the use of multiple heterogeneous NoSQL systems in a distributed environment. They define the conceptual elements necessary for data modeling and identify optimized data distribution patterns.

- In Ref. 25 we show the possibilities of integration of relational and graph databases with the help of a functional data model and its formal language - a typed lambda calculus [language of terms (LT)]. Relations and directed graphs are modeled as sets of functions (single- or multi-valued). Comparing to the previously mentioned abstract models, here we have, moreover, a powerful extendible query language LT at disposal. Data selection is performed in the source systems using native query languages (SQL and Cypher), and then the results are mapped onto data structures associated with the source query term. Clearly, the approach requires a user-friendly version of LT. All other data manipulation functions, e.g. data integration of separated source systems, are executed on data functions.

- A product combining the scale and flexibility of NoSQL with the safety and data integrity of relational systems is FaunaDB. ${ }^{\mathrm{n}}$ Its authors call their approach relational NoSQL databases. Their DBMS manages any data (relational, document, graph and temporal) via the API (GraphQL, FQL, etc.) that best meets user needs. ACID transactions are also guaranteed. Fauna Query Language (FQL) is another example of a functional query language used in the context of NoSQL and relational DB.

\subsubsection{Ontology integration}

The problem of uniform data access to the semantic heterogeneity of source systems is often based on ontologies. A more advanced integrating architecture including several NoSQL databases is proposed in Ref. 26. The databases are described by several ontologies and a generated global ontology. Global SPARQL queries are transformed into query languages of sources. Another representative of this approach is Tatooine, ${ }^{27}$ a lightweight data integration prototype, which allows to enrich structured text, modeling Parliament debates, with metadata about the people who speak in these debates. RDF simplifies the creation of integration links between datasets.

\section{Conclusions}

The purpose of this work was to show that it is not possible to use simply traditional approaches for the integration of RDBMSs and NoSQL database. The reason is the complementarity of these database types. We focused on various approaches to integration of these heterogeneous databases. Due to this heterogeneity, new

${ }^{\mathrm{n}}$ https://fauna.com/faunadb. 
problems occur in practice. NoSQL databases are flexible but their data design requires also significant modeling decisions that impact their performance in integrated architectures. ${ }^{24}$

Particularly, these approaches are influenced by the fact that Big Data and Big Analytics are considered. In practice, the key issues for building Big Data processing infrastructure are in decisions concerning NoSQL databases. They include, in particular,

- choosing the right (correct) product,

- designing a suitable database architecture for a given application class.

For example, we choose the key-value store if there is a need to manage simple schema and faster read/write with no frequent updates. A document store is appropriate if we need a flexible schema with complex querying. Column stores are good for extreme write speeds with relatively less velocity reads. And graph databases are better for applications requiring traversal between data nodes where there is a need to store properties of each data node as well as the relationships between them.

However, the role of a person is also significant especially in Big Analytics. Currently, the DM process is driven by an analyst or data scientist. Depending on the application scenario, the person determines a portion of the data from which, for example, useful patterns can be extracted. A better solution would, however, be to have an automated DM process in place to get approximate synthetic information about both structure and content of large amounts of data. This is still a big problem for Big Data analysts.

Current challenges for DB research in data integration include:

- developing meaningful and usable formalisms for modeling NoSQL databases and a sufficiently general user-friendly query language;

- modeling multimodel databases including relational and NoSQL ones in one infrastructure;

- improving the quality and scalability of DM methods. Interpreting a query especially in the schema's absence - and received answers may be nontrivial.

- transforming content into a structured format for later analysis, because many data today is not natively in a structured format. At the same time, with a filtering we can reduce the volume of data.

Finally, we would like to emphasize that integration of NoSQL and relational DBs is only a part of more general data integration activities in practice. Considering these DBs in a cloud environment we can observe, in the last years, attempts to achieve even a cloud integration. Cloud integration is then the act of combining different cloud-based systems into an integral whole using mediation or federation.

\section{Acknowledgments}

This work was supported by the Charles University Project Q48. 


\section{References}

1. M. Stonebraker, D. Deng and M. L. Brodie, Database decay and how to avoid it, in Proc. 2016 IEEE Int. Conf. Big Data (IEEE, 2016), pp. 7-16.

2. J. Pokorný, Big Data storage and management: Challenges and opportunities, in ISESS 2017: Environmental Software Systems: Computer Science for Environmental Protection, IFIP Advances in Information and Communication Technology, Vol. 507 (Springer, 2017), pp. 28-28.

3. J. Pokorný, Integration of relational and NoSQL databases, in ACIIDS 2018: Intelligent Information and Database Systems, Lecture Notes in Computer Science, Vol. 10752 (Springer, 2018), pp. 35-45.

4. X. Wang, H. Zhang, J. Wang and Y. Zhou, Sentiment analysis of name entity for text, in Proc. 28th Int. Conf. Software Engineering and Knowledge Engineering (2016), pp. $37-41$.

5. A. Corbellini, C. Mateos, A. Zunino, D. Godoy and S. Schiaffino, Persisting Big-Data: The NoSQL landscape, Inf. Syst. 63 (2017) 1-23.

6. N. Marz and J. Warren, Big Data: Principles and Best Practices of Scalable Real-Time Data Systems, 1st edn. (Manning Publications Co., 2015).

7. J. Pokorný, NoSQL databases: A step to databases scalability in Web environment, Int. J. Web Inf. Syst. 9(1) (2013) 69-82.

8. J. Pokorný, Graph databases: Their power and limitations, in Proc. 14th Int. Conf. Computer Information Systems and Industrial Management Applications, eds. K. Saeed and W. Homenda, Lecture Notes in Computer Science, Vol. 9339 (Springer, 2015), pp. 58-69.

9. E. Meijer and G. M. Bierman, A co-relational model of data for large shared data banks, Commun. ACM 54(4) (2011) 49-58.

10. A. Abelló, Big Data design, in Proc. 11th Int. Workshop Data Warehousing and OLAP (ACM, New York, 2015), pp. 35-38.

11. Á. Vathy-Fogarassy and T. Hugyák, Uniform data access platform for SQL and NoSQL database systems, Inf. Syst. 69 (2017) 93-105.

12. P. J. Sadalage and M. Fowler, NoSQL Distilled: A Brief Guide to the Emerging World of Polyglot Persistence (Pearson Education, Inc., 2013).

13. F. R. Oliveira and L. M. del Val Cura, Performance evaluation of NoSQL multi-model data stores in polyglot persistence applications, in Proc. 20th Int. Database Engineering \& Applications Symp. (2016), pp. 230-235.

14. G. Zhao, Q. Lin, L. Li and Z. Li, Schema conversion model of SQL database to NoSQL, in Proc. 9th Int. Conf. P2P, Parallel, Grid, Cloud and Internet Computing (IEEE, 2014), pp. $355-362$.

15. C. Chasseur, Y. Li and J. M. Patel, Enabling JSON document stores in relational systems, in Proc. 16th Int. Workshop Web and Databases (2013), pp. 1-6.

16. J. Lu, I. Holubova and B. Cautis, Multi-model databases and tightly integrated polystores: Current practices, comparisons, and open challenges, in Proc. 27th ACM Int. Conf. Information and Knowledge Management (ACM, 2018), pp. 2301-2302.

17. C. Bondiombouy and P. Valduriez, Query processing in multistore systems: An overview, Research Report No. RR-8890, INRIA Sophia Antipolis - Méditerranée (2016).

18. Ch. Li and J. Gu, An integration approach of hybrid databases based on SQL in cloud computing environment, Softw. - Pract. Exp. 49(3) (2019) 401-422.

19. V. Herrero, A. Abelló and O. Romero, NoSQL design for analytical workloads: Variability matters, in ER 2016: Conceptual Modeling, Lecture Notes in Computer Science, Vol. 9974 (Springer, 2016), pp. 50-64. 
20. G. A. Schreiner, D. Duarte and R. dos Santos, SQLtoKeyNoSQL: A layer for relational to key-based NoSQL database mapping, in Proc. 17th Int. Conf. Information Integration and Web-based Applications \& Services (ACM, New York, 2015).

21. O. Curé, R. F. Hecht, Ch. Le Duc and M. Lamole, Data integration over NoSQL stores using access path based mappings, in DEXA 2011: Database and Expert Systems Applications, Lecture Notes in Computer Science, Vol. 6860 (Springer, 2011), pp. 481-495.

22. W.-Ch. Chung, H.-P. Lin, S.-Ch. Chen, M.-F. Jiang and Y.-Ch. Chung, JackHare: A framework for SQL to NoSQL translation using MapReduce, Autom. Softw. Eng. 21 (2014) 489-508.

23. F. Bugiotti, L. Cabibbo, P. Atzeni and R. Torlone, Database design for NoSQL systems, in ER 2014: Conceptual Modeling, Lecture Notes in Computer Science, Vol. 8824 (Springer, 2014), pp. 223-231.

24. M. Hewasinghage, N. B. Seghouani and F. Bugiotti, Modeling strategies for storing data in distributed heterogeneous NoSQL databases, in Proc. Int. Conf. Conceptual Modelling, Lecture Notes in Computer Science, Vol. 11157 (Springer, 2018), pp. 488-496.

25. J. Pokorný, Integration of relational and graph databases functionally, arXiv:1809.03822 [cs.DB].

26. O. Curé, M. Lamole and Ch. Le Duc, Ontology based data integration over document and column family oriented NoSQL, arXiv:1307.2603 [cs.DB].

27. R. Bonaque, T.-D. Cao, B. Cautis, F. Goasdoué, J. Letelier, I. Manolescu, O. Mendoza, S. Ribeiro, X. Tannier and M. Thomazo, Mixed-instance querying: A lightweight integration architecture for data journalism, in Proc. VLDB Endow. 9(13) (2016) 1513-1516. 